\title{
CAMBIOS DE TIEMPO Y ESPACIO/ CAMBIOS SOCIALES, BAJO EL IMPACTO DE LA MODERNIZACIÓN
}

\author{
ELLEN WOORTMANN \\ Universidade de Brasília
}

Resumen: Este articulo tiene como objec tivo discutir la relación entre espacio, tiempo e género en el contexto de comunidades pescadoras del Nordeste de Brasil. Centra-se en el a nalizis de essas c ateg oria s hac ia la ótic a femenina, destac ando las transformac iones negativa soc urridas en el universo de género face a la modernización en general y el turismo en especial.

Palabras claves: género; tiempo; espacio; comunidades pescadoras; turismo.

Este tra bajo tiene por objetivo a na lizar la relación entre el espacio, la construcción de género y la condic ión femenina en comunida desque se identific an como "pesca doras".

Los datos empíricos resultan del trabajo de campo realizado en el litoral de Rio Grande do Norte. El proceso de cambio a quí a na liza do debe servisto como una tendencia general que alcanza los poblados de aquel litoral de manera diferenciada en el tiempo. Así, en a lgunos, el proceso estaba más avanzado, sin embargo otros permanecían, al tiempo de la investigación, próximos a una situación tradicional. ${ }^{1}$

Cuando se habla de comunidades pescadoras, imagínase, muy frecuentemente, actividades productivas y agentes sociales relacionados apenas a la pesca. Por otro la do, los estudios relativos a esas comunidades tienden a privilegia r el punto de vista del hombre, o sea, del pescador. No obstante, no es incomún que en esas comunidades haya también a gricultura, a demás de la pesca, como es el caso de los grupos estudiados por Peirano ${ }^{2}$ en Ceará y por Beck $k^{3}$ en Sta Catarina, Dantas Cameiro ${ }^{4}$ y Maldonado en

Copyright $(2006$ by Revista Estudos Feministas.

${ }^{1}$ Esta es una versión reducida del trabajo Degradación ambiental/degradación social femenina, no publicado, 51 ojas, presentado en el 120 Intemacional Economic History Congress, en Madrid, 1998. Agradezco a las alumnas de postgrado colombianas Laura Ordoñes y Silvia Monrou por su colaboración con el trabajo y a Kla as Woortmann por la crítica al texto y por sus sugerencias.

2 Mariza PEIRANO, 1975.

${ }^{3}$ Ana M. BECK, 1981.

${ }^{4}$ Simone DANTAS CARNEIRO, 1979.

${ }^{5}$ Simone MADONADO, 1991. 
Pa ra iba, o de los grupos estudia dos por Fa ris; ${ }^{6}$ Nemec $;{ }^{7}$ O mohund ro ${ }^{8}$ en Ca na dá. En va rios grupos, como el estudiado por Maués ${ }^{9}$ y aquellos que son el foco de este trabajo, ${ }^{10}$ la agricultura es pensada como actividad femenina. Privilegiar el punto de vista masculino sería olvidar las a ctividades a gríc olas que constituyen el dominio de las mujeres.

La conjugación de planos del discurso y de autoridad, masculino y femenino, público y privado, deriva del que Cronin"1 llamó de "armonía entre ideales culturales y sistema productivo". En este trabajo procuro privilegiar el punto de vista femenino. Busco mostrar cómo el tiempo y el espacio son construídos por las mujeres; cómo estas dos categorías están entrelazadas una con la otra, cómo se relacionan con la condición femenina y cómo la mujer se (re) construye en el tiempo por el espacio.

La clasificación del espacio natural es también una clasificación de espacios sociales y de dominios pertinentes a cada género. En un plano más general, el mares percibido como dominio del hombre, en oposición a la tierra, dominio de la mujer. No obstante, esa clasific ación bipolar se relativiza y se descompone en otras oposiciones de menor escala. El mar se subdivide en mar de afuera, mar alto, o denso, espacio del trabajo masculino por excelencia, y en mar de dentro (entre la playa y los arrecifes) donde tanto hombres como mujeres ejercen actividades productivas. La tierra, a su vez se subdivide tradicionalmente entre el espacio de la agricultura y la playa, el primero concebido como esencialmente femenino, y el segundo como un espacio intermedio donde, tal como en el mar de dentro, trabajan tanto mujeres como hombres. El espa cio, por ende, no permaneció siempre el mismo, aunque en el plano del discurso público la oposición Tierra/Mar, como espacios de género, tienda a mantenerse.

En la perspectiva aquí adoptada, la condición femenina se (re) construye en el tiempo, y por el espacio. Así, la construcción del tiempo es también la construcción del género, pues él es percibido a tra vés de experiencias que son específic as a ca da género, en espacios que les son también específicos. Si el tiempo y el espacio son categorías universales del pensamiento, son también categorías pensadas culturalmente; cada sociedad los piensa a su manera, según su cultura y su historia particulares. Por otro lado, cada sociedad es constituida por (y constituye) personas diferenciadas, localiza das en relaciones de género - dimensión que aquí me interesa - de clases sociales, etc; y localizadas también en las rela ciones entre sus luga res y otros luga res.

Las mujeres, porejemplo, perciben la llegada del alambre de púascomo el fin de sus tierras de cultivo, o sea, del espa cio fundamental de su identidad. Y a los hombres los perciben como el comienzo de la caña de azúcar, para ellos un nuevo espacio y una nueva altemativa de trabajo, a unque negativamente valoriza da. La s mujeres no dejan de incluir en su concepción del tiempo el espacio de los hombres, el mar. Lo hacen de manera contrastiva, pues el mar permaneció inmutable, mientras la tierra se transformó. Los hombres, a su vez, no dejan de incluir la tierra en concepción del tiempo, pero ese se construye fundamentalmente por lo que pasa en el mar.

Las personas son históricas, y por eso mismo su concepción del tiempo, hoy, probablemente no es la misma de ayer. Antiguamente, con el contenido que tiene hoy, sólo existe hoy. La percepción del tiempo histórico es, ella misma, histórica, pues es dada

\footnotetext{
${ }^{6}$ J a mes FARIS, 1972.

7 Thoma s NEMEC, 1972.

8 J ohn T. OMOHUNDRO, 1985.

${ }_{9}$ Maria Angélic a MAUÉS, 1977.

${ }^{10}$ Ellen WOORTMANN, 1992.

${ }^{11}$ Constance CRONIN, 1977
} 
en un momento específico de la historia - y no menos histórica es, porcierto, la percepción del antropólogo que habla sobre el tiempo de los otros; no hace mucho tiempo, los antropólogos no se preocupaban en distinguir la temporalidad de mujeres y de hombres.

Las mujeres perciben el tiempo de la manera como lo hacen ahora porque están colocadas en un momento puesto porla historia. El tiempo pone, entonces, las condiciones de su propria representación: para ser constituído por el pensamiento, él constituye el pensamiento. La representación del tiempo es, pues, el resultado de una temporalidad.

Pa ra las mujeres estudiadas, lo antiguamente parece ser un tiempo congelado "para atrás", iniciándose el movimiento a partir de una ruptura "para el frente". En la percepción de las mujeres con quien hablé, la memoria comienza con un pasado que "siempre fue", para aproximarse de un presente que no se debería hacer.

El tiempo es percibido por las mujeres a través de pares de oposiciones antiguamente era un tiempo de abundancia, de los manglares sin polución, un tiempo de respeto, un tiempo en que "se vivía como gente". Hoy, tiempo extraño, es un tiempo de precisión, de violencia resultante de la ambición, un tiempo en que "se mora que nem caranguejo".

En el pasado idealiza do y realizado no había violencia contra las mujeres. Los pescadores, cuando regresaban del mar, siempre beben, y siempre bebían. En el pasa do, sin embargo, "los hombres bebían mucho pero no tocaban ni un pelo de las mujeres"... "Mi padre bebió toda su vida, pero nunca nos levantó la mano cuando niñas, ni contra mamá". La violencia que existía en el pasado era entre hombres, por efecto de disputas, o como acto colectivo entre quien infring iese las reglas morales, como en el caso de un hombre, de fuera de la comunidad, linchado por haber robado pescado de su compadre, un crimen menos contra un individuo que contra valores centrales del grupo. La violencia de hoy contra las mujeres, percibida como un hecho nuevo, es atribuída por ellas a la ambición que habría atacado a los hombres, pero es también atribuída a la pérdida de los espacios productivos tradicionales de la mujer, pues fue esa pérdida que las hizo parecer "perezosas", y que condujo a la pérdida del respeto. Vale notar que hoy la violencia, inclusive contra las mujeres es un acto individual (que puede inclusive ser condenado por la colectividad), mientras que en el pasado representado era un acto colectivo que podía ir del linchamiento hasta un equivalente del charivari europeo.

Las mujeres ta mbién miden el tiempo de manera relativa, específic a a su género de un lado, es medido porgeneraciones de mujeres: "en el tiempo de mi madre"; "en el tiempo de mi abuela", cuando se refieren a épocas no vividas porquien está hablando y, notablemente, cuando hablan de espacios que hoy no existen más. De otro lado, el tiempo es referido al ciclo de vida de la mujery, de cierta forma, a supropio cuerpo. Los acontecimientos son localizados en el tiempo con referencia a los nacimientos de los hijos. Así, el alambre de púas llegó a una localidad, del punto de vista de una mujer, "después que nació J oão; otro evento ocurrió "poco a ntes de nacer María". Del punto de vista de otra mujer, el alambre de púas llegó cuando "yo estaba esperando a Antonio". El alambre de púas es un evento que marca la historia, por la supresión de sus espacios productivos; delimitando espacios, delimita ta mbién tiempos. Pero el a la mbre de púas es a su vez marcado por el ciclo de vida de la mujer. Si una mujer no sabía cuando algo ocurrió, recurría a otra, que localizaba el evento con relación su propia sucesión de partos, o a otras crisis de vida, como matrimonios y muertes: "fue cua ndo yo me casé"; "fue cuando papá murió". O entonces, "fue cuando mamá se casó", cuando el acontecimiento es transmitido de una generación a otra. Para que yo tradujera esa percepción del tiempo en aquella que es familiar a nosotros, tuve que transformar la 
temporalidad vivida de aquellas mujeres en nuestra cronología abstracta a prisionando el tiempo en fechas y décadas.

Para localizara lgo en el tiempo, la s mujeres recorren, pues, a su cic lo de vida. Pero para pensarse, las mujeres construyen el tiempo a través del espacio.

La construcción del tiempo es a náloga a la construc ción del espacio: en un primer momento el espacio se constituye por una gran oposición - mar-tierra - que corresponde a la oposición hombre-mujer; en un segundo momento, otros espacios son incluídos, con una atenuación de oposiciones. Con relación al tiempo ocurre algo semejante: se pasó de una primera posición - antiguamente/hoy - para la inclusión de momentos intermediarios, como mediando los polos extremos del tiempo que, no obstante, son marcados por la presencia/ausencia del espacio fundamental para la mujer.

Es importante observar que el tiempo, así como el espacio, son producidos a lo largo de varias expresiones sucesivas que componen el "encuentro etnográfico", y por ta nto, a lo largo de otro tiempo. Depende entonces del contexto de la conversación, que se puede militaro no al discurso público.

El tiempo, por otro lado, no fue hablado por las mujeres como algo en sí, mas que para hablar/pensarsobre ellas mismas. No se habló sobre el tiempo pero sobre lasmujeres a través del tiempo. Para verse en el presente, ellas se ven en el pasado. Es como si el tiempo fuera un espejo que muestra una imagen invertida, puespresente y pasado tienen señales opuestas. En el caso, pa rafra seando la conocida imagen a ntropológica, el tiempo es mirror for women. El tiempo, relacionado a la representación que hacen las mujeres sobre sí mismas, surge a través del espacio. Hace un tiempo que hubo en el pasado lo antiguamente, tiempo en que no se tocaba ni un pelo de la mujer. Era sobretodo el tiempo en que existía un espacio, las sueltas, una época que "siempre fue así", que marca el contraste con el presente, en la relación con los hombres. Trabajadoras en el pasado; "perezosas" hoy. Respetadas en el pasado; agredidas hoy.

El tiempo, en cualquier lugar, puede ser construído de diferentes maneras, pues él es siempre contextual. No creo que se pueda hablar sobre las concepciones. Así mismo las mujeres pueden percebir el tiempo de distintas maneras, dependiendo de lo que tienen en vista. El tiempo histórico a quí considerado no es el único tiempo que perciben. Existe también, y hoy más que ayer, el tiempo diario, en el cual alquilan sus variadas a c tividades, a sí c omo a quellas de sus hijos. Desde este punto de vista, las mujeres "tienen menos tiempo" que los hombres. En el contexto de las relaciones de género, sin embargo, el tiempo históric o es pensa do básicamente a tra vés del espa cio, y este es un espacio de género. La abundancia se derivaba de la existencia de las sueltas; el respeto se debía al hecho de las mujeres ejercer el cultivo en esas mismas tierras sueltas.

Se puede decirque el tiempo histó ric o, irreversible, es c onstruíd o por substra c c iones. Antiguamente, era un tiempo marca do por la experiencia del espacio, fundamental para la mujer, y el movimiento temporal es percibido porla pérdida sucesiva de ese y de otros espacios donde se hacía la actividad femenina. Es interesante observar, por ejemplo, que el manglar sólo fue referido cuando se habló de su pérdida, sea por la privatización o por la contaminación. También sólo se habló de las sueltas para resaltar su desaparecimiento. Si el tiempo es una manera de pensarse, el espacio también lo es, pues cada espacio es un dominio, y con el transcurrir del tiempo, del punto de vista femenino, fue diferente para mujeres y hombres. Para las mujeres, el tiempo transcurró porla pérdida de espacios específic os que ella sartic ula ban en un espacio total. El espa cio de los hombres, sin embargo, permaneció contante, pues, si la tierra se tomó cautiva, el mar continúo tierra libre. Para ellos no hubo pérdida del espacio; lo que ocurrió fue un 
cambio de los hombres dentro del mismo espacio, alcanzados por la ambición. Lo que ya es otro componente de la percepción: en lo que concieme a los hombres (vistos por las mujeres), permaneció el espacio y cambió la natura leza intrínseca de la persona; en lo que concieme a las mujeres, permaneció su naturaleza y cambió el espacio.

Además del tiempo irreversible, existe otro, reversible, o cíclic o. En las palabras de las mujeres ese tiempo es referido al espacio de los hombres: es el tiempo dado por la conjugación entre la natura leza del mary las activida des de los hombres. De modo general, el año se divide entre el tiempo de pesca y otro de no-pesca, así como en períodos definidos por la especificidad de la actividad pesquera. Tal como el espacio y el tiempo histórico, también el tiempo cíclico se constituye inicialmente por una gran oposición, enseguida mediatizada por otras menores. Se podría también especular sobre si en el pasado no existieron dos tiempos cíc lic os complementarios: un, referido a la tierra y a la agricultura, y otro referido al mar y ala pesca, aunque este último fuera hegemónico y constitutivo del discurso público. Es posible también que en el pasado del tiempo cíclico que se repite siempre igual, fuera dominante, en la medida en que el pasado fuera un tiempo de reproducción simple, fundada en la predominancia del valor de uso, y un tiempo de histoire immobile. Tendríamos posiblemente una aproximación con el tipo de percepción del tiempo a naliza da por Bourdieu ${ }^{12}$ con relación a campesinos de Argelia, antes del "desencantamiento" de su mundo por la historia traída por el colonizador. Las nociones del tiempo histórico y tiempo cíclico se aproximan de aquellas de tiempo estructural y tiempo ecológico formuladas por Evans-Pritc hard..$^{13}$ Por tiempo ecológico él se refiere a secuencias temporarias que emergen de la relación con el a mbiente natural, no como imposición inmediata de la naturaleza, sino como representación socialmente construída, porque, en términos soc ia les, no existe una na tura lezen sí, sino una natura leza cultura Imente a prendida.

Las variaciones productivas del tiempo ecológ ico implic an el reordenamiento a lo largo del año de las relaciones con la naturaleza, y de las relaciones sociales en ellas envueltas. No me interesa aquí analizar esa dimensión cíclica que fue analizada por Caldas Britto ${ }^{14}$ con relación a otros pescadores brasileños.

La noción de tiempo estructural se refiere a una manera de pensar el tiempo, basada en puntos de referencia que poseen significado para determinados grupos, proyectando en el pasado relaciones sociales del presente.

El tiempo estruc tural sería entonces relaciona do a la identidad del grupo. El énfasis de mi estudo sugiere, sin embargo, que si hay puntos signific a tivos de la historia percibida accionados para la construcción de la identidad del grupo - y como fue visto, el todo es frecuentemente confundido con los hombres - los hay ta mbién para la constitución de la identidad de género.

Las mujeres se ven frente a los hombres en un proceso que transita de la complementariedad para la dependencia, en la medida en que en el tiempo se substra en espacios. En esa perspectiva, los marcos temporales son marcos de la transformación del género que sólo existe frente al otro género. El otro contrastante construído por el tiempo/ espacio no es otro grupo, sino otro género del mismo grupo, asi como las personas de hoy de aquel mismo lugar, porla proyección en el pasado de la experiencia del presente. La alteridad, aquí, emerge del tiempo.

\footnotetext{
12 Pierre BOURDIEU, 1977.

${ }^{13}$ Evans EVANS-PRITC HARD, 1972.

${ }^{14}$ Rosyan CALDAS BRITTO, 1989.

${ }^{15}$ In WOORTMANN, 1992.
} 
Perel espacio de las mujeres no es a pena suna ca teg oría cultural del pensa miento. Él es también un ambiente. Este ambiente es un espacio total compuesto por espacios espec ífic os a rtic ula dos entre sí por las a c tivida des de las mujer. Los da tos etnog rá fic os ${ }^{15}$ revelan que la mujerno sólo a ric ula relacionessociales, sino también relacionesespaciales. La historia de esas mujeres es, en buena parte, la historia de la desarticulación de esos espacios.

Si el espacio es un a mbiente, un ecosistema, él no es a penas un a mbiente na tural, dado, más un ambiente cognitivamente aprendido y culturalmente construído. Como ambiente construído, es un espacio "significado", cuyo uso social le a tribuye un sentido. La noción de a mbiente incluye, entonces, las relaciones sociales y la cultura que hacen de la "población" de esse ecosistema una sociedad. Si la historia es dada por la desarticulación del ambiente construído, ella es también el proceso de atribución de nuevos signific ados al espacio, de nuevos usos sociales, y del desplazamiento social de los agentes tradicionales. El cambio ambiental signific a la alteración de las relaciones con el esapcio, de los hombres entre sí, y de lass mujeres con los hombres en función de ese espacio. Si el espacio verifica los marcos de referencia que poseen significado, constituyendo un tiempo estructural, la historia, desde el punto de vista femenino, es el encogimiento y la degradación del espacio/ambiente.

El tiempo ecológico fue como que invadido por el tiempo histórico. Con las transformaciones ocurridas, un nuevo ritmo cíclico se está yuxtaponiendo a aquel constituído por la interacción con el mar. Se trata del ritmo impuesto por la presencia intensa de veraneantes y turistas. Estos son, a hora, parte del ecosistema, si lo conceb imos no apenas como relaciones naturales sino también espaciales. Además de una cosecha de pescados, existe también una "safra" de turistas, y estos contribuyeon, para modificar la condición social de la mujer, además de haber altera do el ciclo a nual de actividades de la comunidad como un todo.

El turismo es parte de un gran movimiento de capital. Vera neantes y turistas, forman parte del proceso global de integración a un nuevo orden económico. De un lado, la industria turística se juntó a la agroindustria en la expropiación de espacios básicos de la mujer. De otro lado, los turistas cambiaron a las personas del lugar. Buscando reencontrar el paraíso perdido en sus ciudades, corren el riesgo de construir el infiemo para los "auténticos" del lugar, repitiendo lo que ya ocurró en otras localidades "paradisía cas". ${ }^{16}$ Pormás "altemativos" que se piensen, son parte del proceso que alteró el movimiento y la organización del espacio/ambiente.

Ese movimiento que cambia el tiempo por cambiar el espacio puede ser visto como la realización en escala local y con su especificidad propia, de la "gran transformación" de que habla Polanyi, ${ }^{17}$ lanzando las personas en un nuevo orden gobemado por los "nexos monetarios". También en esos lugares se inventó el mercado, en la medida en que la tierra y el trabajo - a los cualespodríamosagragarlas casa de las familias locales - ganan nuevos significados bajo la forma de mercancía. Comenzando con el enclosure de las antiguas sueltas y pasando por la privatiza ción (que se agrega a la contamina ción) de losmang la res; por la creciente hegemonía de la captura de langosta sobre la pesca; por la transformación de la pesca a través de nuevas tecnologías, y con ellas, sino la transformación del espacio del mar, en el espacio del mar; y por el turismo, la antigua simetría y autarquía relativas, condición de la reciprocidad, del valor de uso y

${ }^{16}$ Cf. Andrea BINDÁ, 1989; CALDAS BRITTO, 1989.

${ }^{17}$ Karl POLANYI, 2000.

${ }^{18} \mathrm{Cf}$. BOURDIEU, 1977. 
de la comunidad, en un tiempo que no se pensaba en economizar, ${ }^{18}$ cedieron lugara la ambición del nuevo tiempo, que es dinero, un tiempo extraño.

A lo largo de ese proceso, las relaciones entre hombres y mujeres se alteraron, como fue visto. De una relación de complementariedad se pasó a una relación de dependencia de las mujeres para con los hombres. Si antiguamente las mujeres eran coresponsables por la abundancia, hoy ellas "comen de la mano del marido", como dijo una esposa del lugar.

Eso levanta una contradicción: la "gran transformación" significó el paso de una agricultura de subsistencia para otra, de mercado, cuando en el esapcio de la roza de las mujeres, dirigida fundamentalmente para el consumo familiar y para circuítos de reciprocidad, fue insta la da la a groindustria según el modelo de plantation. Las tra dicionales balsas fueron siendo sustituídas por las embarcaciones a motor, aumentando la produc tividad del trabajo. En a mboscasosocurrió una expansión de la sfuerzas produc tivas. La captura de langosta trajo consigo un nuevo "ethos" de trabajo, con la ind ividualización y el espíntu de ganancia monetaria. El "nexo monetario" pasó a gobemaresferas crecientes de las relaciones sociales. En resumen, a quellas conunidades pasaron por un proceso de modemización, transitando de la Gemeinschaft para la Gesellschaft, del status para el contrato, de lo tradicional a lo modemo. Sin embargo, esa modemización, en el ámbito local, no trajo consigo la "emancipación de la mujer", como debería ocurrir según una teoría más general. Por el contrario, trajo la dependencia y la desvalorización de la mujer.

La continuidad de la historia podrá deshacer lo paradójico estableciendo nueva coherencia entre rela ciones de producción y condición femenina. Supone la teoría general que el ingreso de la mujer en el mercado de trabajo tra e consigo su liberación, pero todo depende de la naturaleza de ese mercado. Hasta ahora, sin embrago, éste no parece ser el caso de la mayoría de las mujeres en las comunida des estudiadas. En ellas, como fue visto, la mujer siempre fue parte integrante de la fuerza de trabajo. No apenas gerenciaba el consumo de la familia, como también realizaba la producción de alimentos y de insumos para la pesca. Se encontraba, por lo tanto, fuera del mercado de trabajo, ya que la fuerza de trabajo no era mercancía. Hoy, ella ya se encuentra en ese mercado; no obstante, se percibe subordinada al hombre.

Mirando su paso, las mujeres ven su condición de entonces como siendo una condición que se podría llamar de complementariedad equilibrada, transformada para la mayoría en dependencia subordinada. Esa transformación resulta de la invasión y polución del espacio/ambiente por fuerzas sociales externas al "mundus" de las comunidades locales. O sea, de la subversión de significado de espacio/ambiente.

Fina Imente, es necesa rio establecer ciertas rela tiviza ciones. Una está relaciona da a propria noción de complementariedad. Considerada desde el punto de vista de la familia, la relación hombre-mujer, cuando ésta es ama de casa apenas, y no productora a utónoma, es también complementario. La actividad de la ama de casa es central para la reproducción de la familia y de la fuerza de trabajo tanto en grupos campesinos como en la clase trabajadora urbana. Además, en el campesinado norteño es el propio honor del padre de familia que depende del no-trabajo de la mujer. Allí es el respeto del punto de vista del hombre que se ve amenazado caso la mujertrabaje, pues el trabajo es una categoría apenas referida al hombre. El equilibrio reposa ahí en la complementariedad entre la roza, espacio masculino, y la casa, espacio femenino, y en la ausencia en la mujer del primero; él depende de la mujer ser ama de casa. La complementariedad es por tanto contextual, y es en el contexto de los grupos aquí estudiados que debe ser visto el pasaje percibido por las mujeres de una complementariedad considerada equilibrada para una dependencia vista como subordinada. 
Los cambios registra dos en aquellas comunidades no signific an el pasaje de una "economía natural" para otra, de mercado. Rela ciones de mercado siempre existieron; lo que se puede deciresque ellas son hoy más importantes que ayer. No se pueden minimizar las transformaciones que hicieron de la tierra y del trabajo mercancías, que produjeron una reorientación del valor de uso para el valorde cambio, a fectando tanto el mundo de los hombres como el de las mujeres. Del mismo modo, las personas no son inmutables, nuea s circ unstanc ia s eng end ran eng end ran nuevas práctic as, nuevas disposic iones y un nuevo habitus, al mismo tiempo estruc turado por ella y estructurante de la historia. ${ }^{19}$

Algunos hombres dejaron de ser pescadores para volverse langosteros, a propiándose así de los nuevos tiempos. Algunas mujeres se volvieron gerentes de negocios familiares dirigidos para el lucro, apropiándose de la nueva "vida" introducida por el turismo en la cotid ia nida d del grupo, o sea, dand o un nuevo sig nific ado al tiempo ec ológ ico o cíclic 0 . Otras, más modestas, se limitan a trabajar como empleadas domésticas. Esa reorientación puede ser pensada como una respuesta social de adaptación al nuevo a mbiente ecológico-social, que es ta mbién un nuevo momento de su historia. Se establece entonces una nueva complementariedad que, en el primercaso tenderá probablemente a acentuar la diferenciación social, pero que, en el segundo caso, que corresponde a la mayoría de las mujeres, no restableció la abundancia ni trajo de regreso el respeto; por el contrario, convive con la violencia contra la mujer.

A pesar de los cambios, hasta ahora estas comunidades continúan pensándose como de pescadores. Su identidad desde este punto de vista, no se alteró. La situación de las mujeres, no obstante, y su auto-imagen cambiaron bastante.

\section{Referencias bibliográficas}

BECK, Ana M. "Roça, pesca e renda". Boletim de Ciências Sociais, n. 23, p. 21-32, 1981. BINDÁ, Andrea H. Nativos e abastados. 1989. Dissertação de Graduação, Departamento de Antropologia, Universidade de Braślia.

BOURDIEU, Pierre. "Reproduction simple et temps cyclique". In: . Algérie 60: strutures éc onomiques et struc tures temporelles. Paris: Éditions de Minuit, 1977. p. 19-43.

CALDAS BRITTO, Rosynan C. Modernidade e tradição. 1989. Dissertação de Mestrado, CPDA Curso de Pós-Graduação em Desenvolvimento Agrícola, Universidade Federal Rural do Rio de Janeiro.

CRO NIN, Constance. "Illusion and Rea lity in Sic ily." In: SC HLEGEL, Alice. Sexual Stratific ation. New York: Columbia Univ. Press, 1977. p. 67-93.

DANTAS CARNEIRO, Simone. Terra liberta. 1979. Dissertação de Mestrado, Departa mento de Antropologia, Universidade de Braślia.

EVANS-PRITC HARD, Evans E. The Nuer. Oxford: Oxford Univ. Press, 1972.

FARIS, J a mes. Cat Harbour: A Newfoundland Fishing Settlement. Toronto: University of To ronto Press, 1972.

MALDONADO, Simone C. O mito da terra liberta. 1991. Tese de Doutorado, Departamento de Antropologia, Universidade de Braślia.

MAUÉS, Maria Angélica. Trabalhadeiras e camarados. 1977. Dissertação de Mestrado, Departamento de Antropologia, Universidade de Brasilia.

${ }^{19}$ BOURDIEU, 1980. 
NEMEC, Thomas. "I Fish With My Brother." In: ANDERSEN, Ra oul. North Atlantic Fishermen. Toronto: Newfoundland Univ. Press, 1972. p. 18-36.

OMOHUNDRO, J ohn T. "One Potato, Two Pota to." Natural History, v. 94, n. 6, 1985. p. 22-29. PEIRANO, Mariza. A reima do peixe. 1975. Dissertação de Mestrado, Departamento de Antropologia, Universidade de Brasilia.

POLANYI, Kart. A grande transformação. Rio de J aneiro: Campus, 2000.

WOORTMANN, Ellen F. "O a mbiente e a mulher: o caso do litoral do Rio Grande do Norte, Brasil". Latin American Studies, Tóquio, n. 12, p. 31-53, 1992.

\section{Changes in Space and Time/Social Change under the Impact of Modernization}

Abstract: The aim of this article is to disc uss the relations between space, time and gender in the context of fishing communities of Northeastern Brazil. Centered on the point of view of women, it calls attention to the negative changes that took place in the female universe as a result of modernization.

Key Words: Gender; Time; Space; Fishing Communities; Modernization. 